\title{
A comparison of dietary patterns of middle aged men in France and Northern Ireland: the PRIME Study
}

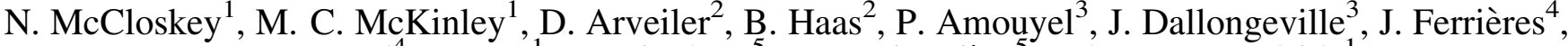 \\ V. Bongard ${ }^{4}$, F. Kee ${ }^{1}$, A. Bingham ${ }^{5}$, P. Ducimetière ${ }^{5}$ and J. V. Woodside ${ }^{1}$ \\ ${ }^{1}$ Centre of Public Health, Queen's University Belfast, Belfast, BT12 6BJ, UK, ${ }^{2}$ MONICA-Strasbourg, France, \\ ${ }^{3}$ MONICA - Lille, France, ${ }^{4}$ MONICA-Toulouse, France and ${ }^{5}$ INSERM U780, Villejuif, France
}

The traditional approach to assess diet has been to examine nutrients or foods in isolation ${ }^{(1)}$. Due to the complexities of dietary intake, this approach has limitations and, more recently, the assessment of dietary patterns has emerged as a complementary approach ${ }^{(1)}$. Dietary patterns represent combinations of food consumption, accounting for cumulative and synergistic effects of nutrients in the diet ${ }^{(2)}$.

The aim of this study was to calculate a priori dietary scores in the PRIME prospective cohort study, which included dietary data on men aged 50-59 years from Belfast, N. Ireland $(n=2748)$, and Lille $(n=2627)$, Strasbourg $(n=2611)$, and Toulouse $(n=2610)$ in France.

Four a priori dietary scores were calculated for each centre based on data from the 22-item FFQ used in PRIME. Scores were calculated as follows: (1) Fruit and Vegetable (FV) variety score - one point awarded for each different FV consumed at least once per week -maximum score of 8 possible. (2) Overall variety Score - one point given for each different food type consumed at least once per week maximum of 16 points possible. (3) Adapted Mediterranean Diet Score (aMDS) - which included fish, fruit, vegetables, nuts, use of polyor mono-unsaturated oils on salad, use of vegetable oils in cooking and alcohol consumption. Points were awarded based on recommended quantities - maximum score of 7 possible. (4) Daily FV score, which quantified total FV consumption in portions per day.

For all a priori scores, the mean value of the French population was significantly higher than the Belfast population $(p<0.001)$. For both the aMDS and Daily FV score, scores differed significantly between centres; Toulouse had the highest scores in both cases, and Belfast the lowest. For the FV variety score, Toulouse and Strasbourg had the highest scores, which were similar; these differed significantly from Lille, which was third, and Belfast fourth, which was significantly different from all three. For the overall variety score, Lille had the highest score, which differed significantly from the Toulouse and Strasbourg centre scores. Belfast again had the lowest score compared to each of the French centres.

\begin{tabular}{lcccc}
\hline Score & Country & Mean Score & SD & $P$ value \\
\hline Adapted MDS & France & 3.15 & 1.19 & $<0.001$ \\
(Max score $=7$ ) & Belfast & 1.78 & 1.13 & \\
FV variety score & France & 6.40 & 1.42 & $<0.001$ \\
(Max score $=8$ ) & Belfast & 5.84 & 1.93 & \\
Overall variety score & France & 11.37 & 2.19 & $<0.001$ \\
(Max score $=16)$ & Belfast & 10.97 & 2.52 & \\
Daily FV score & France & 2.53 & 0.18 & $<0.001$ \\
(Portion/day) & Belfast & 2.07 & 0.19 & \\
\hline
\end{tabular}

$P$ values from independent samples t-test. ANOVA and SNK test performed to examine centre differences.

In conclusion, the French population had higher scores for 'Overall variety' and 'FV variety', as well as higher daily FV consumption and a higher aMDS score, indicating closer resemblance to the Mediterranean diet. These differing dietary patterns may have different implications for disease risk. Further work will assess whether these dietary scores are related to risk of cardiovascular disease in the PRIME study.

1. Hu FB (2002) Curr Opin Lipidol 13, 3-9.

2. Jinlin F, Binyou W \& Terry C (2007) J Postgrad Med 53, 139-43. 